\title{
EDITORIAL
}

\section{Type A behaviour and ischaemic heart disease ${ }^{1}$}

It is a common medical belief that psychosocial factors, in some form or another, contribute to the risk of developing ischaemic heart disease (IHD). Anecdotal evidence comes from many of the great figures of medical history. William Harvey described a coronary patient whose illness was the result of 'anger and indignation which he yet communicated to no one' (Leibowitz, 1970). John Hunter's angina was brought on by 'agitation of the mind' and he was reported to have died from an attack provoked by a particularly irritating hospital board meeting in 1793 (Home, 1794). In 1910, Osler described the typical coronary patient as 'the keen and ambitious man, the indicator of whose engine is always at full speed ahead' - a description very like that which has latterly been termed Type A behaviour.

Since Osler, there have accumulated many clinical and epidemiological reports relating psychosocial factors to ischaemic heart disease (e.g. see the reviews by Jenkins, 1971, 1976). These data are of varying quality and do not provide consistent evidence of a role in aetiology. Perhaps the strongest evidence for a causal link between psychosocial factors and ischaemic heart disease comes from the studies of Type A behaviour (Dembroski, 1977). We would do well, therefore, to examine critically the evidence on Type A behaviour with a number of questions in mind. Has a causal link been established? What is the magnitude of the association? To what extent do variations in the frequency of Type A behaviour account for variations in the occurrence of IHD?

First, what is Type A behaviour? One might have hoped for a definition - which we do not have. It is described as an 'action-emotion' complex (Rosenman, 1977) that is characterized by impatience, a sense of time-urgency, competitiveness, striving for achievement, aggressiveness, hyper-alertness, restlessness, explosive speech and abruptness of gesture. The techniques used for measurement do not clarify the issue very much. In their initial cross-sectional studies, Friedman and Rosenman used clinical judgements to classify people and demonstrated an association between Type A behaviour and ischaemic heart disease in both men and women (Friedman \& Rosenman, 1959; Rosenman et al. 1961).

Subsequently, in a longitudinal study in California, they used a more formal version of clinical judgement. Type A behaviour was assessed by a structured interview. In classifying people, importance was placed not only on the content of the answers to the questions but on the individual's mannerisms and behaviour during the interview. This technique had the curious property that exactly $50 \%$ of the population at entry into their longitudinal study were classified as Type A, there being no reason a priori why this should be so. It is not clear which characteristics of Type A individuals should be considered as representing the crucial part of the behaviour pattern and which are merely associated characteristics. Nevertheless, this global classification into Type A or Type B was remarkably revealing. Over an $8 \frac{1}{2}$ year follow-up period in the California study, Type A men had twice the incidence of ischaemic heart disease as Type B men (Rosenman et al. 1975).

For these studies to be replicated, reproducible measures of Type A behaviour had to be developed. Jenkins developed an elaborate questionnaire designed for self-administration - the Jenkins Activity Survey (JAS) - which was correlated with the structured interview (73\% agreement) (Jenkins et al. 1967). Although the JAS assessment of Type A behaviour has been shown to be associated with IHD in cross-sectional studies (Jenkins, 1976), in Rosenman \& Friedman's longitudinal study the JAS was less strongly associated with IHD incidence than was the structured interview (Jenkins et al. 1974). It seems that the JAS is missing some crucial component of the Type A behaviour pattern - either because of the way the questions were asked, or the way the questionnaire was scored, or possibly

\footnotetext{
1 Address for correspondence: Dr Michael Marmot, Department of Medical Statistics and Epidemiology, London School of Hygiene and Tropical Medicine, Keppel Street, London WC1E 7HT.
}

0033-2917/80/2828-5660 \$01.00 ㄷ 1980 Cambridge University Press 
because the subjects' mannerisms and style displayed in the structured interview are not well correlated with the answers to the questionnaire, but are correlated with the risk of disease.

Other approaches to measuring Type $\mathrm{A}$ behaviour have been made. In the Framingham Massachusetts Heart Study, a Type A scale was constructed from 10 questions that asked about presumed Type A characteristics. Men and women classified as Type A on this questionnaire have an increased prevalence and incidence of ischaemic heart disease (Haynes et al. 1978, 1980). In the UK, yet a fourth measure of Type A behaviour, the Bortner scale, has been shown in a case-control study to be associated with IHD (Heller, 1979). Other studies, while not specifically measuring Type A behaviour, have produced data consistent with the above (Jenkins, 1976).

The consistency of these results, while not clarifying our understanding of Type A behaviour, do increase the likelihood that it reflects characteristics that are causally related to ischaemic heart disease. There is some evidence both from an autopsy study (Friedman et al. 1968) and from studies of patients undergoing coronary angiography (Zyzanski et al. 1976; Blumenthal et al. 1978) that Type A behaviour may be related to the underlying process of atherosclerosis, i.e. to the chronic disease process.

There are plausible biological reasons why Type A behaviour might precipitate an acute myocardial infarction or sudden death. Type A subjects have been reported to have a faster clotting time than Type B (Friedman \& Rosenman, 1959), increased platelet aggregation (Jenkins et al. 1975) and a higher urinary output of catecholamines during working hours (Friedman, 1977a) - although this latter finding has not been confirmed (DeBacker et al. 1979). Whether the association between IHD death and Type A behaviour (Friedman 1977b) is related to these mechanisms is not clear.

In the California study, Type A behaviour was related to new cases of myocardial infarction, to angina pectoris and to recurrent ischaemic heart disease. It does appear that the differences in risk of clinical IHD between Type As and Type Bs cannot be explained by differences in serum cholesterol, or blood pressure or in cigarette smoking (Rosenman et al. 1975). In other words, the epidemiological and clinical/laboratory evidence suggests that Type As may possibly be at higher risk because of a direct effect on the neuroendocrine system.

These studies have all been directed at discriminating one individual's disease risk from another's. A different kind of question is the extent to which variations in the frequency of Type A behaviour may explain group or population differences in the rate of occurrence of IHD.

It would indeed be an intriguing notion if, as has been suggested (Rosenman, 1977), the twentiethcentury coronary epidemic is the result of an environment that has increased the prevalence of Type $A$ behaviour; or if males have more IHD than females because of their greater tendency to be Type A; or if Italians, French and Japanese have low IHD rates because of their relative infrequency of Type A behaviour; or if clerical officers in the British civil service have a higher risk of IHD than their administrative superiors (Marmot et al. 1978) because the administrators are less likely to be Type A.

These are not easy questions to assess, first because only the most enthusiastic supporter of the Type A concept would suggest that all hitherto unexplained variations in the occurrence of IHD may be explained by variations in one single behaviour pattern. To put it in perspective, the risk of developing IHD in Type A individuals was marginally less than twice the risk in Type Bs after adjustment for other risk factors. Secondly, the testing of these assertions presupposes the existence of a measure of Type A behaviour that is not culturally, socially or sexually biased. The structured interview was developed for use in a study of employees of several corporations in California and, as Jenkins (1976) points out, initially most studies using the JAS did not include persons with low education, rural occupations, or aged over 65 . Only one or two studies have included women. To show that men tend more often to be Type A than women (Waldron et al. 1977) or that Americans tend more to be Type A than Belgians (Kittel et al. 1978) is suggestive, but one would like to have confidence that the measure reflects the same thing in both sexes or cultures. For example, the JAS was used in a study of Japanese-Americans (Cohen, 1974). Their low Type A scores were consistent with their low rates of IHD compared with white Americans, but a factor analysis of the answers to the JAS questionnaire revealed that the questions clustered together in a different manner from that observed in the study 
of predominantly middle-class white Californians. There was the clear implication that the questionnaire responses did not mean the same thing in these two different cultures.

One approach to the cross-cultural validation of a Type A measure is to test predictive validity. If a measure of Type A behaviour is shown to be associated with IHD in different cultures, it increases one's confidence in the measure, although it does not necessarily imply that one may then use this measure to compare the frequency of Type A behaviour. A high/low dichotomy may successfully discriminate IHD cases from non-cases in different cultures, without the absolute levels of the scores having the same meaning. Nevertheless, there is evidence that Type A behaviour is associated with IHD in populations outside North America (Heller, 1979; Zyzanski, 1977).

We are thus at an interesting stage in the research on Type A behaviour. The balance of evidence points to a causal association with ischaemic heart disease, but the origin and nature of Type A behaviour have been described in only the vaguest terms. Attempts to demonstrate a dose-response relationship or to show that components of the Type A behaviour pattern are related to ischaemic heart disease have proved largely unsuccessful (Jenkins et al. 1967). More intensive research is now under way, aimed at better description of Type A behaviour.

It seems likely that further advances in our knowledge will come from studies now in progress that are attempting to relate Type A behaviour to risk of IHD and to other psychosocial factors in subjects other than middle-class white American men. It has been suggested that Type A behaviour is neither a personality pattern nor a reflection of a stressful environment, but a combination of the two. It may be that Type A behaviour is a middle-class American way of reacting. Reactions to a stressful environment in women, in working-class Englishmen, in Belgians or Swedes may take a form other than that typical of Type A behaviour, but the underlying notion still may be correct; i.e. that a failure to cope in an appropriate manner with a stressful environment predisposes to ischaemic heart disease. We need answers to the questions of what constitutes a 'stressful environment' or an appropriate manner of coping. The results of the research on Type A behaviour and ischaemic heart disease make it clear that it is worth seeking answers to these questions.

\section{MICHAEL MARMOT}

\section{REFERENCES}

Blumenthal, J. A., Williams, R. \& Kong, Y. (1978). Type A behaviour and angiographically documented coronary disease. Circulation 58, 634-639.

Cohen, J. B. (1974). Sociocultural change and behaviour patterns in disease etiology: an epidemiologic study of coronary disease among Japanese Americans. Unpublished doctoral dissertation: University of California, Berkeley.

DeBacker, G., Kornitzer, M. \& Kittel, F. (1979). Relation between coronary-prone behaviour pattern, excretion of urinary catecholamines, heart rate, and heart rhythm. Preventive Medicine 8, 14-22.

Dembroski, T. M. (ed.) (1977). Proceedings of the Forum on Coronary-Prone Behaviour. DHEW Publication no. (NIH) 78-1451. Washington, D.C.

Friedman, M. (1977a). Type A behaviour pattern: some of its pathophysiological components. Bulletin of the $\mathrm{New}$ York Academy of Medicine 53, 593-604.

Friedman, M. $(1977 b)$. Type A behaviour: its possible relationship to pathogenetic processes responsible for coronary heart disease (a preliminary inquiry). In Proceedings of the Forum on Coronary-Prone Behaviour (ed. T. M. Dembroski), pp. 179-184. DHEW Publication no. (NIH) 78-1451. Washington, D. C.

Friedman, M. \& Rosenman, R. H. (1959). Association of specific overt behaviour pattern with blood and cardiovascular findings. Journal of the American Medical Association 169, 1286-1296.

Friedman, M., Rosenman, R. H. \& Straus, R. (1968). The relationship of behaviour pattern $A$ to the state of the coronary vasculature: a study of fifty-one autopsy subjects. American Journal of Medicine 44, 525-537.

Haynes, S. G., Feinleib, M. \& Levine, S. (1978). The relationship of psychosocial factors to coronary heart disease in the Framingham study. II. Prevalence of coronary heart disease. American Journal of Epidemiology 107, 384402.

Haynes, S. G., Feinleib, M. \& Kannel, W. B. (1980). The relationship of psychosocial factors to coronary heart disease in the Framingham study. III. Eight-year incidence of coronary heart disease. American Journal of Epidemiology 111, 37-58.

Heller, R. F. (1979). Type A behavio ar and coronary heart disease. British Medical Journal ii, 368.

Home, E. (1794). A short account of the author's life by his brother-in-law, Everard Home. In Cardiac Classics (ed. F. A. Willius and T. E. Keys), pp. 269-275. C. V. Mosby: St Louis (1941)

Jenkins, C. D. (1971). Psychologic and social precursors of coronary disease. New England Journal of Medicine 284, 244-255, 307-317.

Jenkins, C. D. (1976). Recent evidence supporting psychologic and social risk factors for coronary disease. New England Journal of Medicine 294, 987-994, 1033-1038.

Jenkins, C. D., Rosenman, R. H. \& Friedman, M. (1967). Development of an objective psychological test for the determination of the coronary-prone behaviour pattern in employed men. Journal of Chronic Diseases 20, 371379.

Jenkins, C. D., Rosenman, R. H. \& Zyzanski, S. J. (1974). Prediction of clinical coronary heart disease by a test for the coronary-prone behavior pattern. New England Journal of Medicine 290, 1271-1275. 
Jenkins, C. D., Thomas, G. \& Olewine, D. (1975). Blood platelet aggregation and personality traits. Journal of Human Stress 1, 34-46.

Kittel, F., Kornitzer, M., Zyzanski, S. J., Jenkins, C. D., Rustin, R. M. \& Degre, C. (1978). Two methods of assessing the Type A coronary-prone behaviour pattern in Belgium. Journal of Chronic Diseases 31, 147-155.

Leibowitz, J. O. (1970). The History of Coronary Heart Disease. University of California Press: Berkeley.

Marmot, M. G., Rose, G., Shipley, M. \& Hamilton, P. J. S. (1978). Employment grade and coronary heart disease in British civil servants. Journal of Epidemiology and Community Health 32, 244-249.

Osler, W. (1910). The Lumleian Lectures on angina pectoris. Lancet i, 697-702, 839-844.

Rosenman, R. H. (1977). History and definition of the Type A coronary-prone behaviour pattern. In Proceedings of the Forum on Coronary-Prone Behaviour (ed. T. M. Dembroski), pp. 13-17. DHEW Publication no. (NIH) 78-1451. Washington, D.C.
Rosenman, R. H. \& Friedman, M. (1961). Association of specific behaviour pattern in women with blood and cardiovascular findings. Journal of the American Medical Association 24, 1173-1184.

Rosenman, R. H., Brand, R. J., Jenkins, C. D., Friedman, M., Straus, R. \& Wurm, M. (1975). Coronary heart disease in the Western Collaborative Group Study. Final follow-up experience of $8 \frac{1}{2}$ years. Journal of the American Medical Association 233, 872-877.

Waldron. I., Zyzanski, S. J., Shekelle, R. B., Jenkins, C. D. \& Tannenbaum, S. (1977). The coronary-prone behaviour pattern in employed men and women. Journal of Human Siress 3, 2-18.

Zyzanski, S. J. (1977). Associations of the coronary-prone pattern. In Proceedings of the Forum on Coronary-Prone Behaviour (ed. T. M. Dembroski), pp. 46-66. DHEW Publication no. (NIH) 78-1451. Washington, D.C.

Zyzanski, S. J., Jenkins, C. D. \& Ryan, T. J. (1976). Psychological correlates of coronary angiographic findings. Archives of Internal Medicine 136, 1234-1237. 